just entering this area of occupational health, and an interchange among the research delivery communities on what else may or may not help and why.

${ }^{1}$ Frida Marina Fischer, ${ }^{2}$ Orna Tzischinsky, ${ }^{3}$ Tamar Schochat, ${ }^{4}$ Lucia Rotenberg, ${ }^{5}$ Kazutaka Kogi, ${ }^{6}$ Stephen Popkin

${ }^{1}$ Dept Environmental Health, School of Public Health, University of São Paulo, São Paulo, Brazil

${ }^{2}$ Emek Yezreel College, Emek Yezreel, Israel

${ }^{3}$ University of Haifa, Haifa, Israel

${ }^{4}$ Laboratory of Health and Environmental Education, Oswaldo Cruz Foundation, Rio de Janeiro, Brazil

${ }^{5}$ Ohara Memorial Institute for Science of Labour, Japan

${ }^{6}$ U.S. Department of Transportation, Volpe Centre, Cambridge, USA

\section{2a MULTIPLE JOB STRESSORS OF NIGHT AND ROTATING SHIFT WORKERS THAT AFFECT HEALTH AND WELLBEING: BASIS FOR COMPREHENSIVE INTERVENTIONS FOR THEIR SOLUTIONS}

${ }^{1}$ Frida Marina Fischer* ${ }^{2}$ Aline Silva-Costa, ${ }^{3}$ Rosane H Griep, ${ }^{3}$ Lucia Rotenberg, ${ }^{4}$ Michael Smolensky. 'Department of Environmental Health, School of Public Health, University of São Paulo, São Paulo, Brazil; ${ }^{2}$ Federal University of Triângulo Mineiro, Department of Collective Health, Uberaba, Minas Gerais, Brazil, ${ }^{3}$ Laboratory of Health and Environment Education, Oswaldo Cruz Institute, Rio de Janeiro, Brazil; ${ }^{4}$ Department of Biomedical Engineering, Cockrell School of Engineering, The University of Texas at Austin, Austin, TX, USA

\subsection{6/oemed-2018-ICOHabstracts.138}

Multiple workplace xenobiotic, physical, and psychosocial factors can affect employee wellbeing and health, particularly when working nights. The human circadian time structure (CTS) is an unappreciated, yet potentially important consideration to establishing threshold limiting values, employee biological monitoring (BM) procedures, and recommended biological exposure indices (BEIs). Encounters by night workers, before CTS adjustment, to potentially harmful workplace contaminants and stressful physical conditions, occur during a different circadian stage than day workers. Numerous animal and human investigations document prominent circadian patterning in the biological tolerance to a broad array of chemical, biological, and physical stressors often found in occupational settings. Time-qualified for biological rhythms reference values, several currently utilised in clinical laboratory medicine, are likely to be of relevance to employee surveillance. Workplace psychosocial factors are additionally of great importance to employee wellbeing, with several demand-control-social support and effort-reward imbalance models serving to assess detrimental outcomes. Despite recognition the psychosocial workplace milieu can affect the physical and psychological health of dayworkers, there is a scarcity of knowledge of its impact on night and shift employees. Some studies indicate low job control, high physical demands, low supervisor social support, and high overcommitment can be more problematic for night than day shift workers. At-work violence is an additional psychosocial stress, with police officers, security personnel, bank employees, professional drivers, and other service employees in regular contact with the public, in particular, at elevated risk. The severity of workplace violence problems and their consequences is probably underestimated, especially when co-existing among stressors with known impact on workers' health. Practical considerations and recommendations for action to mitigate the effect of these multiple job stressors of particularly high relevance to night and shift workers are presented.

\section{$1602 \mathrm{~b}$ SLEEPINESS AND VIGOUR IN AIRLINE GROUND CREW SHIFT WORKERS MOVING FROM 8- TO 12-HOUR ROTATING SHIFT SCHEDULES: A PILOT FIELD STUDY}

${ }^{1}$ Orna Tzischinsky ${ }^{*},{ }^{2}$ Satanai Hadish-Shogan, ${ }^{2}$ Tamar Shochat. ${ }^{1}$ Emek Yezreel College, Emek Yezreel, Israel; ${ }^{2}$ Cheryl Spencer Department of Nursing, Faculty of Social Welfare and Health Sciences, University of Haifa, Haifa, Israel

10.1136/oemed-2018-ICOHabstracts. 1382

Objective To investigate the effects of transition from 8 - to 12 hour shifts on sleepiness and vigour of employees during morning and night shifts.

Methods Thirty-nine airline ground crew shift workers (mean age $38.9 \pm 8.2 \mathrm{y}$; mean seniority $13.9 \pm 7.1 \mathrm{y}, 19$ male) volunteered in a pilot study. During each round, employees were assessed during one morning and two night shifts, by completing a sleepiness questionnaire (the Karolinska Sleepiness Scale, KSS) hourly, and a vigour questionnaire $(3$ times in the 8 hour, 4 times in the 12 hour rounds) per shift. Repeated measures ANOVA was performed to compare changes in sleepiness and vigour levels in 8 hour and 12 hour shift plans, during day and night shifts.

Results For morning shifts main effects were found for sleepiness $\left[\mathrm{F}_{(3,58)}=13.1, \mathrm{p}<0.001\right]$ and for shift plan $\left[\mathrm{F}_{(1,18)}=7.4\right.$, $\mathrm{p}<0.014]$. Sleepiness levels rose throughout both morning shifts, and were lower during the 12 hour than during the 8 hour shifts. Furthermore, main effects were found for vigour $\left[\mathrm{F}_{(1,25)}=11.37, \mathrm{p}<0.001\right]$ and for shift plan $\left[\mathrm{F}_{(1,18)}=14.87\right.$, $\mathrm{p}<0.001]$. Vigour levels decreased in both morning shifts, and were higher during 12 hour shifts than in the 8 hour shifts. No interactions were found.

For night shifts main effects were found for sleepiness $\left[\mathrm{F}_{(7,18)}=9.9, \quad \mathrm{p}<0.001\right], \quad$ and for sleepiness"shift plan $\left[\mathrm{F}_{(3,81)}=3.2, \mathrm{p}<0.023\right]$. During the 8 hour round, sleepiness consistently increased with a slight decrease towards the end of the shift, while in the 12 hour round sleepiness increased until 04:00 am, after which there was a decrease and again and increase towards the end of the shift.

Furthermore, main effects were found for vigour $\left.\left[\mathrm{F}_{(1,25)}=11.37, \mathrm{p}<001\right]\right)$, and for shift plan $\left[\mathrm{F}_{(1,18)}=14.87\right.$, $\mathrm{p}<0.001$ ], during the 12 hour shift round participants' vigour levels were higher than in the 8 hour shift round. Vigour levels decreased during the night in both rounds, no interaction was observed.

Conclusion An overall improvement was observed in somnolence and vigour indices of employees after the transition to 12 hour shift, which appears to be preferable over the 8 hour shift.

\section{C SLEEPY ON THE NIGHT SHIFT? BIO-PSYCHO-SOCIAL FACTORS OF SUBJECTIVE SLEEPINESS IN FEMALE NURSES DURING THE NIGHT SHIFT}

${ }^{1}$ Tamar Shochat* ${ }^{*}{ }^{1,2}$ Nataly Zion. ${ }^{1}$ Cheryl Spencer Department of Nursing, Faculty of Social Welfare and Health Sciences, University of Haifa, Haifa, Israel; ${ }^{2}$ Bnei Zion Medical Centre, Haifa, Israel

10.1136/oemed-2018-ICOHabstracts.1383 
Introduction Sleepiness during work hours is the most common complaint of night shift workers, and is a sensitive indicator of performance decrements. Sleepiness levels vary between individuals, yet few have investigated individual factors as predictors of night shift sleepiness, and these have shown mixed results. We aimed to examine the effects of biopsycho-social factors on subjective sleepiness of nurses during the night shift.

Methods Female nurses $(n=119)$ working irregular rotating shifts were recruited from two hospitals in Northern Israel, using convenience sampling by clusters. Inclusion criteria were working at least $75 \%$ of full time, with at least one night shift per week. Exclusion criteria were pregnancy, a diagnosed sleep disorder, and/or chronic medical conditions. Subjective sleepiness was measured hourly during two night shifts using the Karolinska Sleepiness Scale (KSS). Sleep was monitored by actigraphy 24 hours before and until the end of the night shifts. Participants completed a socio-demographic questionnaire, the Munich ChronoType Questionnaire for Shiftwork (MCTQ $^{\text {shift) }}$, the Pittsburg Sleep Quality Index (PSQI) and the Pre-Sleep Arousal Scale (PSAS).

Results Mixed models stepwise analyses found main effects for hour, age, cognitive pre-sleep arousal and number of children on nighttime sleepiness (all $\mathrm{p}<0.01$ ). Effects of chronotype on sleepiness were inconsistent. Interactions were found for age *number of children $(\mathrm{p}<0.01)$, pre-sleep cognitive arousal*chronotype $(\mathrm{p}<0.05)$, and age* chronotype $(\mathrm{p}=0.06)$. Older nurses were less sleepy than younger nurses, but this impact was attenuated by early chronotype and having more children. High cognitive pre-sleep arousal, but not sleep, predicted increased sleepiness, especially in nurses with late chronotype. Discussion The impact of bio-psycho-social factors on night shift sleepiness is complex and depends on mutual interactions between these factors. Nurses who are young, late chronotypes and with high cognitive pre-sleep arousal require special attention and support, and must develop personal strategies for maintaining vigilance on the night shift.

\section{2d BREAST CANCER AND SHIFT WORKING IN A LIGHT POLLUTED WORLD}

Abraham Haim*, Zubidat Abed. The Israeli Centre for Interdisciplinary Research in Chronobiology, University of Haifa, Haifa, Israel

\subsection{6/oemed-2018-ICOHabstracts.1384}

Introduction Breast cancer (BC) is increasing worldwide together with light pollution (LP) emerging from various outdoor and indoor sources. Results of different studies including our research centre report on the relations between BC-incidences and exposure to Artificial Light at Night (ALAN). The trend for energy saving-ALAN increases the problem, as light intensity is increasing and mainly that of short wavelength (SWL), within the blue part of the spectrum $(450-500 \mathrm{~nm})$. Our master biological clock, located in the hypothalamus, entrained by light/dark cycles is in charge of our temporal organisation from cell functions. It is not only light-intensity, which changes with the 24 hour cycles, but also the dominant parts of the light spectrum, which reach's earth. Those dominant parts, signalling for daytime are the SWL, including those between $450-500 \mathrm{~nm}$ a range known as an efficient suppressor of the nocturnal pineal produced hormone Melatonin (MLT). We attempted to study the nexus: ALAN, MLT-
Suppression, epigenetic modifications and BC-cells proliferation in subcutaneously inoculated female mice.

Methods Mice were acclimated for two weeks under 8L:16D, at a constant ambient temperature testing various sources of illumination differing in spectrum composition. After inoculation, we exposed mice to ALAN of the same illumination of daytime. We measured the following variables: Body mass, tumour volume, MLT-production and levels of Global DNA methylation (GDM) levels.

Results We revealed the existence of the proposed nexus. Response to ALAN is depended on the wavelength illumination source. SWL-illumination bulbs as white-LED or compact florescent have a higher negative effect compared with that of incandescent or carbon bulbs. We emphasise a relation between tumour volume, level of MLT-suppression and GDMlevels.

Discussion We suggest that human populations under increasing LP-levels of SWL-illumination are in a high risk for becoming BC-patients, it should be of great interest to set the threshold for exposure to SWL-illumination and BC-risk.

\section{2e CAN ON-SHIFT NAP BENEFIT NIGHT WORKERS' HEALTH? STUDIES ON BLOOD PRESSURE AND OBESITY IN NURSING TEAMS}

${ }^{1}$ Lucia Rotenberg*, ${ }^{2}$ Aline Silva-Costa, ${ }^{1}$ Rosane $\mathrm{H}$ Griep. 'Laboratory of Health and Environmental Education, Oswaldo Cruz Foundation, Rio de Janeiro, Brazil, ${ }^{2}$ Department of Collective Health, Federal University of Triângulo Mineiro, Uberaba, Minas Gerais, Brazil

\subsection{6/oemed-2018-ICOHabstracts. 1385}

Introduction On-shift napping can benefit night workers as regards sleep deprivation, adjustment of circadian rhythms, and alertness. But possible benefits of nap on health outcomes are scarcely investigated. Considering that night work is pointed as a risk factor for hypertension and obesity, we investigated the possible attenuation of blood pressure and body mass index increase by on-shift napping among nursing teams.

Methods A cross-sectional study was conducted in a public hospital in Rio de Janeiro, Brazil with nursing professionals who were informally allowed to nap for up to three consecutive hours during working nights. Current and accumulated doses of night work (NW) were studied through the number of working nights/2 week-span and years of $\mathrm{NW}$, respectively. Four outcomes were measured using standard equipment and techniques: systolic blood pressure (SBP), diastolic blood pressure (DBP), hypertension (SBP $\geq 140 \mathrm{mmHg}$ or DBP $\geq 90$ $\mathrm{mmHg}$ or prescription of antihypertensive medication), and body mass index (BMI). The associations between exposure variables and outcomes were based on logistic regressions (hypertension) and generalised linear models (SBP, DBP and BMI).

Results Among non-nappers (but not among nappers), current doses of NW (number of working nights) was significantly associated with increased SBP ( $\beta$-value $=1.39 ; 95 \% \mathrm{CI}$ : 0.31 to 2.49 ) and DBP ( $\beta$-value $=0.80 ; 95 \% \mathrm{CI}$ : 0.10 to 1.50$)$, as well as increased odds for hypertension $(\mathrm{OR}=3.35$; 95\% CI: 1.74 to 6.57). This association was not observed for accumulated doses of NW. As regards BMI, both current and accumulated doses of NW were significantly associated with increased BMI levels $(\beta$-value $=0.364 \quad[95 \%$ CI: 0.002 to 0.749$]$ and $\beta$ value $=0.092$ [95\% CI: 0.011 to 0.173$]$, respectively) only among non-nappers. 